\title{
əThe Influence of the Spectral Truncation on the Simulation of Waves in the Tropical Stratosphere
}

\author{
T. R. Krismer, M. A. Giorgetta, And J. S. vOn StORCH \\ Max Planck Institute for Meteorology, Hamburg, Germany \\ I. FAST \\ German Climate Computing Centre (DKRZ), Hamburg, Germany
}

(Manuscript received 20 August 2014, in final form 23 March 2015)

\begin{abstract}
Convectively triggered waves are the main driver of the tropical stratospheric circulation. In atmospheric models, the model's resolution limits the length of the simulated wave spectrum. In this study, the authors compare the tropical tropospheric wave sources, their projection on the wave field in the lower stratosphere, and the circumstances of their upward propagation in the atmospheric model ECHAM6 with three spectral truncations of T63, T127, and T255. The model internally generates the quasi-biennial oscillation (QBO), which dominates the variability in the tropical stratosphere. This analysis focuses on two opposite phases of the QBO to account for the influence of the background wind field on the wave filtering. It is shown that, compared to the high-resolution model versions, the $\mathrm{T} 63$ version has less convective variability and less wave momentum in the lower stratosphere at wavenumbers larger than 20 , well below the version's truncation limit. In the low-resolution version, the upward propagation of the waves is further hindered by the highly active (relative to the high-resolution versions) horizontal diffusion scheme. However, even in the T255 version of ECHAM6, the convective variability is too small compared to TRMM observations at periods shorter than 2 days and wavelengths shorter than $1000 \mathrm{~km}$. Hence, to model a realistic tropical wave activity, the convective parameterization of the model has to improve to increase the day-to-day precipitation variability.
\end{abstract}

\section{Introduction}

Atmospheric waves drive stratospheric circulations such as the quasi-biennial and the semiannual oscillations in the tropical stratosphere (Holton and Lindzen 1972; Dunkerton and Baldwin 1991; Manzini et al. 1997) and the Brewer-Dobson circulation [Cohen et al. (2014), and references therein]. The waves transport momentum upward, away from their tropospheric sources, and accelerate the mean flow where they dissipate because of longwave radiative damping or wave breaking. Research on the

○ Denotes Open Access content.

Corresponding author address: Marco Giorgetta, Max Planck Institute for Meteorology, Bundesstr. 53, 20146 Hamburg, Germany.

E-mail: marco.giorgetta@mpimet.mpg.de momentum transport by atmospheric waves got much attention in relation to the quasi-biennial oscillation $(\mathrm{QBO})$. The dissipative processes are enhanced in the regions of strong vertical shear associated with the QBO jets, where the full spectrum of equatorial waves, from planetary Kelvin and Rossby waves to mesoscale gravity waves, is necessary to propagate the QBO jets against the resistance of the tropical upwelling (Dunkerton 1997; Sato and Dunkerton 1997). How the stratospheric wave activity distributes among the wave spectrum is still unclear. In the tropics, the contributions from large-scale, equatorial Kelvin and Rossby waves can be derived from radiosonde and satellite observations (Yanai and Maruyama 1966; Ern and Preusse 2009; Ern et al. 2014). However, because of the short spatial and temporal scales of gravity waves, they can only be observed during temporally limited field campaigns (Alexander et al. 2004). Hence, general circulation models are a convenient tool to study the interactions of waves with the large-scale circulation. 
Kawatani et al. (2010) and Evan et al. (2012) analyzed the tropical wave field with high-resolution models resolving waves with wavelengths as short as 200 and $100 \mathrm{~km}$, respectively. They showed that the whole spectrum of resolved waves contributes substantially to the forcing of the mean flow. However, we found that, in a general circulation model spectrally truncated at T63, most of the resolved wave forcing is caused by waves with wavelengths larger than $2000 \mathrm{~km}$, and the forcing decreases quickly with decreasing wavelength (Krismer and Giorgetta 2014).

The comparison of the results from Kawatani et al. (2010) and Evan et al. (2012) to Krismer and Giorgetta (2014) shows that, at a given wavenumber, the momentum that the waves transport depends on model resolution. In this study, we show how wave generation and propagation in the equatorial region differ in three model versions of the atmospheric model ECHAM6 (Stevens et al. 2013) with spectral truncations of T63, T127, and T255, respectively. We show that, at wavenumbers that all model versions resolve, the spectral distribution of precipitation, which is a proxy for convective wave sources, the momentum carried by resolved waves, and the resulting forcing in the tropical stratosphere are resolution dependent. Because atmospheric waves are filtered by the mean flow, we discuss the spectra during two well-defined phases of the quasi-biennial oscillation, when the profiles of the zonal-mean zonal wind in the stratosphere are relatively similar in all model versions.

The most important source of tropical waves is the latent heat release within convective clouds [Fritts and Alexander (2003), and references therein]. Salby and Garcia (1987), Bergman and Salby (1994), and Ortland et al. (2011) showed that the wave momentum flux in the lowermost stratosphere depends on the magnitude, depth, and shape of the latent heating within convective clouds. Above the source level, radiative and diffusive wave damping reduces the strength of the waves while they propagate upward. Longwave radiation damps large-scale waves (Zhu 1993; Ern et al. 2009; Krismer and Giorgetta 2014), and, in most spectral models, a horizontal diffusion scheme damps small-scale waves to avoid the accumulation of energy at wavenumbers close to the truncation limit. Thus, horizontal diffusion lowers the effective resolution of the model (Takahashi 1999).

In this paper, we compare the tropical wave sources and the stratospheric wave filtering in the three model versions truncated at T63, T127, and T255 to explain how the strength of resolved tropical waves depends on spectral resolution.

The paper is structured as follows. In section 2, we describe the ECHAM6 model and the three versions we use. To stabilize the climate in all three model versions, the convective parameterization had to be retuned.
Hence, in section 3, we discuss the strength of the convective wave sources with respect to resolution and tuning. Further, we compare the efficiency of wave damping at different scales and the wave forcing of the QBO jets in the three model versions. We summarize the results and draw conclusions in section 4 .

\section{Model description}

We conduct three Atmospheric Model Intercomparison Project (AMIP)-type simulations with the ECHAM6 atmospheric global climate model (GCM; Stevens et al. 2013) using spectral truncations at T63 (Giorgetta et al. 2012), T127, and T255 and an associated Gaussian grid of approximately $1.9^{\circ}, 0.9^{\circ}$, and $0.4^{\circ}$ resolution, respectively. Following the labeling policy of the Max Planck Institute, which hosts ECHAM6, we refer to these three model versions as E6-MR for T63, E6-HR for T127, and E6-XR for T255. AMIP is designed to force atmospheric models with observed monthly mean global sea surface temperatures and sea ice distributions and with standardized values of the solar constant and atmospheric $\mathrm{CO}_{2}$, ozone, and aerosol concentrations (Gates 1992). Our forcing covers the period from 1979 to 2008.

The vertical grid of all model versions has 95 hybrid sigma pressure levels, which resolve the atmosphere from the surface up to the center of the uppermost layer at $0.01 \mathrm{hPa}$. The top-of-the-model pressure is defined as $0 \mathrm{hPa}$. The grid has a nearly constant vertical resolution of $700 \mathrm{~m}$ from the upper troposphere to the middle stratosphere, and the resolution is better than $1 \mathrm{~km}$ at the stratopause. The version truncated at wavenumber 63 is the atmospheric part of the Max Planck Institute Earth System Model (MPI-ESM; Giorgetta et al. 2013). A number of recent publications based on E6-MR and MPI-ESM review the dynamics of the middle atmosphere (Schmidt et al. 2013), the resolved wave forcing and seasonal modulation of the quasi-biennial oscillation (Krismer and Giorgetta 2014; Krismer et al. 2013), the stratosphere-troposphere coupling (Tomassini et al. 2012), tropical precipitation (Crueger et al. 2013), and model tuning (Mauritsen et al. 2012). Hertwig et al. (2014) compared the global climate in the AMIP runs with E6-MR, E6-HR, and E6-XR, which we also analyze here.

To parameterize the effect of subgrid-scale waves, ECHAM6 includes the scheme for orographic gravity waves described by Lott (1999) and the Hines scheme (Hines 1997a,b) for nonorographic gravity waves. To improve the representation of the polar jets, the orographic gravity waves in E6-HR and E6-XR are 50\% and $75 \%$ weaker than those in E6-MR, respectively. 
In the Hines scheme, the prescribed gravity wave sources are constant in time, the launch level is set to $700 \mathrm{hPa}$, and the source spectrum follows the standard setting of the middle atmosphere ECHAM5 model (MAECHAM5; Manzini and McFarlane 1998; Manzini et al. 2006). However, in E6-MR, the otherwise globally uniform wave-induced horizontal wind perturbations, referred to as rms winds, increase linearly from 1 to $1.2 \mathrm{~m} \mathrm{~s}^{-1}$ from $10^{\circ}$ to $5^{\circ} \mathrm{N}$ and from $10^{\circ}$ to $5^{\circ} \mathrm{S}$. From $5^{\circ} \mathrm{S}$ to $5^{\circ} \mathrm{N}$, the rms winds are constant at $1.2 \mathrm{~m} \mathrm{~s}^{-1}$. Effectively, this modification increases the upward transport of wave momentum. The rms winds in the tropical belt are reduced from $1.2 \mathrm{~m} \mathrm{~s}^{-1}$ in E6-MR to 1.05 and $1 \mathrm{~m} \mathrm{~s}^{-1}$ in E6-HR and E6-XR, respectively. The intention of the reduction of the rms winds was to compensate for the expected increase of resolved wave forcing with increasing resolution and to obtain a realistic period of the quasi-biennial oscillation, which all three models internally generate (Hertwig et al. 2014). E6-MR and E6HR simulate a range of QBO periods between 22 and 34 months and a mean period of 29 months. These numbers are in good agreement with observations (Baldwin et al. 2001). In E6-XR, however, the mean QBO period is only 18 months, which suggests that the parameterized wave forcing is still too large (Scaife et al. 2000; Giorgetta et al. 2006). Because of the different tuning of the Hines scheme, we cannot draw sound conclusions on QBO dynamics from the existing runs, and the computational resources for E6-XR are exhausted. However, for the analysis of resolved wave propagation in the tropical stratosphere, we have to account for the phase of the QBO. The QBO phase determines the zonal wind profile and, thus, the levels where strong wave dissipation occurs. Hence, we compare the wave propagation during two opposite QBO phases, when the zonal wind profiles and, thus, the vertical distribution of critical levels, are similar in all three model versions.

The gravity wave parameterization does not influence the resolved wave-mean flow interactions. The zonal wind perturbations, which the Hines parameterization induces, are two orders of magnitude smaller than the background zonal wind. Thus, they cannot change the vertical shear substantially on the 10-day time scale of the resolved wave dissipation.

The source spectrum of resolved waves depends on the latent heat release computed by the parameterization of stratiform and convective clouds. However, the cloud parameterizations also influence the biases of the global radiation balance. To limit these biases, the cloud mass flux above the nonbuoyancy level is 0.23 in E6-MR and E6-HR and 0.16 in E6-XR. The conversion rate from cloud water to rain is $2 \times 10^{-4}$ in E6-MR, $1.5 \times$ $10^{-4}$ in E6-HR, and $1.2 \times 10^{-4}$ in E6-XR. Without

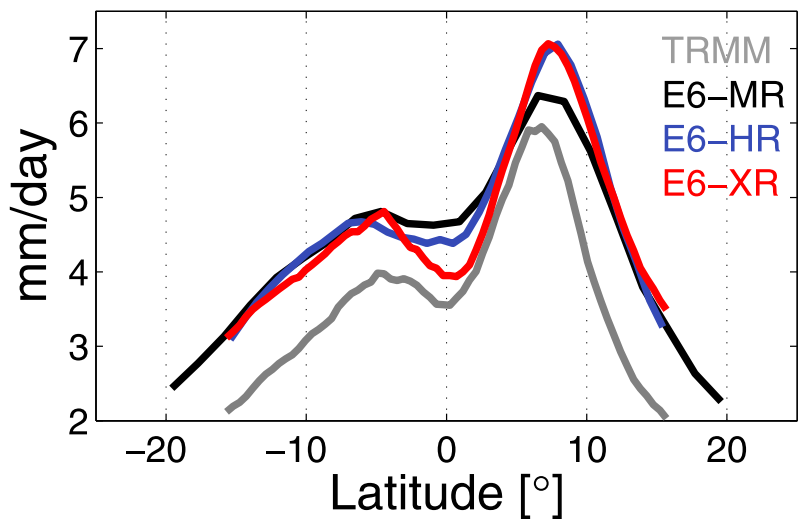

FIG. 1. Longitudinal mean precipitation as a function of latitude in E6-MR, E6-HR, E6-XR, and TRMM, averaged from 2000 to 2003

additional resolution, these measures lead to more and thicker boundary layer clouds and a longer lifetime of convective clouds (Mauritsen et al. 2012). Below, we discuss the influence of the different cloud parameters on the resolved wave field.

In the model, longwave radiation and diffusion damp the resolved waves away from their sources (Krismer and Giorgetta 2014). The setup of the radiation scheme is equal in all three model versions. In the implicit horizontal diffusion scheme, the $e$-folding damping time for the largest wavenumber is $7 \mathrm{~h}$ in E6-MR, $1.5 \mathrm{~h}$ in E6-HR, and $0.5 \mathrm{~h}$ in E6-XR. The diffusion scheme has the eighth order in E6-MR and the sixth order in E6-HR and E6$\mathrm{XR}$. A detailed discussion of the effect of these changes follows below.

\section{Results}

\section{a. Precipitation variability}

In the tropics, the heating within the, mostly convective, clouds is the main wave source [Fritts and Alexander (2003), and references therein]. This heating rate is a source term in the temperature tendency equation of ECHAM6 that was not stored for the simulations conducted with E6-HR and E6-XR. We thus analyze the rain rates as a proxy for the latent heat release.

Figure 1 shows the mean precipitation over the equatorial region in E6-MR, E6-HR, E6-XR, and the Tropical Rainfall Measuring Mission (TRMM; NASA; Huffman et al. 2007) as a function of latitude, averaged from 2000 to 2003. In the Southern Hemisphere, all model versions overestimate the zonal-mean precipitation by about $1 \mathrm{~mm} \mathrm{day}^{-1}$ compared to TRMM, which is between $25 \%$ and $50 \%$ of the TRMM values. In the Northern Hemisphere, E6-HR and E6-XR have more precipitation than 
a)

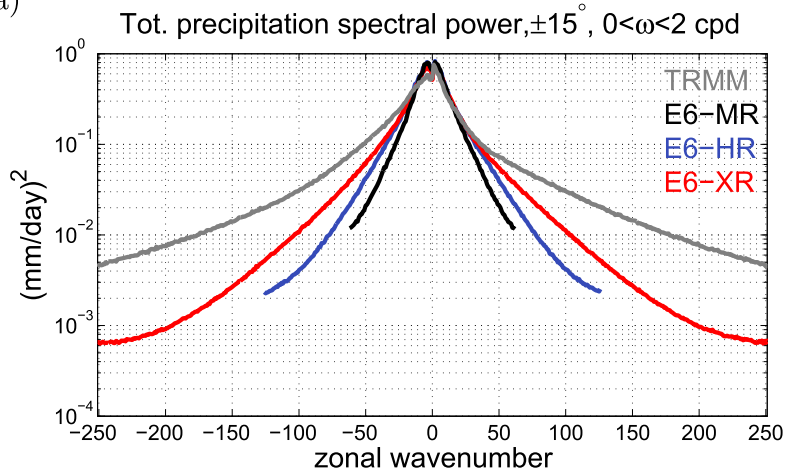

b)

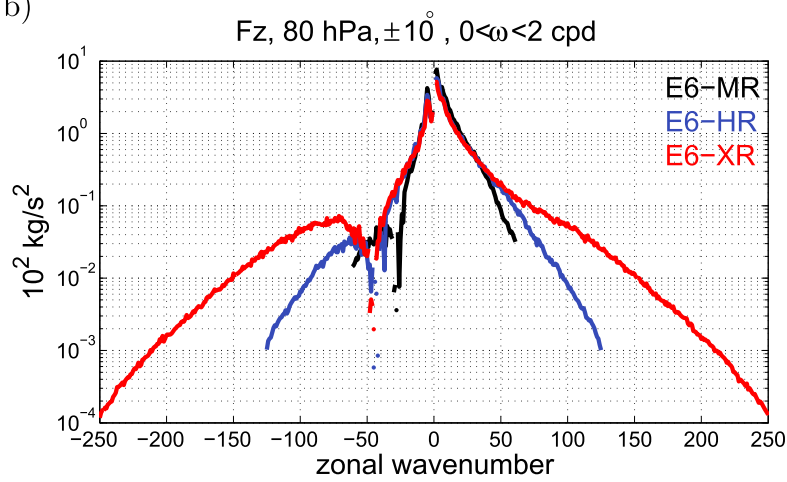

FIG. 2. (a) Variance of the total precipitation $\left(\mathrm{kg}^{2} \mathrm{~s}^{-2}\right)$ and (b) absolute vertical EP flux $\left|F_{z}\right|$ at $80 \mathrm{hPa}\left(\mathrm{kg} \mathrm{s}^{-2}\right)$ averaged over $\pm 10^{\circ}$ of latitude as a function of the zonal wavenumber in E6-MR (black), E6-HR (blue), E6-XR (red), and TRMM (gray). The spectra are averages over overlapping windows including 120 days in (a) and each individual month from 2000 to 2003 in (b). Positive and negative wavenumbers indicate westerly and easterly waves, respectively. Spectral peaks associated with the daily cycle are excluded.

E6-MR, and they again overestimate the zonal-mean precipitation by about $25 \%$ compare to TRMM. However, wave generation is determined not by the mean precipitation but by its spectral variability. Figure $2 \mathrm{a}$ shows the wavenumber spectrum of the tropical precipitation variance in E6-MR, E6-HR, E6-XR, and TRMM. The spectra result from a fast Fourier transform in longitude and time at each latitude over 120-day windows with a 75-day overlap in the period from 2000 to 2003. The values in Fig. 2 are the integrals over all frequencies and the average over $\pm 10^{\circ}$ of latitude. The integrals exclude the spectral peaks of the daily cycle. The TRMM data are interpolated from the original product resolution to the $0.5^{\circ}$ grid of E6-XR. Table 1 shows the numeric values integrated over sets of wavenumbers.

Up to wavenumber \pm 20 , the spectral power of precipitation is approximately equal in E6-MR, E6-HR, and E6-XR (Fig. 2a and Table 1). At wavenumbers larger than \pm 20 , the precipitation variability increases with spectral resolution (Fig. 2a). At wavenumbers between \pm 20 and \pm 63 and at wavenumbers larger than \pm 63 , E6-HR has only about $82 \%$ and $45 \%$ of the spectral power of E6-XR, respectively, and E6-MR has only around $45 \%$ and $24 \%$ of the spectral power of E6-XR, respectively, as Table 1 shows.

The precipitation variability in all model versions compares well to TRMM at wavenumbers lower than \pm 20 . However, the decrease of spectral power with increasing wavenumber is stronger than in TRMM in all model versions (Fig. 2a), although the rate weakens with increasing resolution. Hence, starting at wavenumbers larger than 30, 40, and 50, E6-MR, E6-XR, and E6-HR, respectively, have less precipitation variability compared to TRMM (Fig. 2a). At wavenumbers between 20 and 63 and larger than 63, the spectral power in TRMM is $40 \%$ and $233 \%$ larger, respectively, than in E6-XR (Table 1). Summed over frequencies larger than $0.5 \mathrm{cpd}$, the model versions underestimate precipitation by at least $50 \%$ over all scales (not shown). Hence, although the models generally overestimate the mean precipitation, they do not model small-scale cloud clusters realistically in the tropical region. This deficiency of precipitation schemes is a major problem of general circulation models. It is related to too-low entrainment rates, which tend to produce weak and constant convection rather than more variable and strong convection. As the tropical precipitation variability is a proxy for the wave sources, we expect that ECHAM6 underestimates the generation of waves with periods shorter than 2 days and wavelengths shorter than $1500 \mathrm{~km}$.

\section{b. Stratospheric wave momentum}

The first step from the convective source spectrum to the stratospheric wave forcing is the projection of the precipitation variability on the Eliassen-Palm flux (EP flux) spectrum in the lower stratosphere. The EP flux is the measure of momentum and heat transported by waves in the transformed Eulerian-mean framework

TABLE 1. Spectral power of total precipitation plus or minus two standard deviations integrated over the respective range of wavenumbers and over all frequencies up to $2 \mathrm{cpd}$ in TRMM, E6-MR, and E6-HR as a percentage of the spectral power in E6-XR. Values are averaged over $\pm 10^{\circ}$ of latitude.

\begin{tabular}{|c|c|c|c|}
\hline & \multicolumn{3}{|c|}{$|k|$} \\
\hline & $0-20$ & $20-63$ & $>63$ \\
\hline TRMM (\%) & $99 \pm 21$ & $140 \pm 24$ & $333 \pm 61$ \\
\hline E6-MR (\%) & $100 \pm 19$ & $59 \pm 9$ & $24 \pm 4$ \\
\hline E6-HR (\%) & $100 \pm 21$ & $82 \pm 12$ & $45 \pm 6$ \\
\hline E6-XR $\left[\left(\mathrm{mm} \mathrm{day}^{-1}\right)^{2}\right]$ & $27.7 \pm 7$ & $11.9 \pm 2$ & $3.5 \pm 0.5$ \\
\hline
\end{tabular}


TABLE 2. Vertical EP flux plus or minus two standard deviations integrated over the respective range of wavenumbers and over all frequencies up to $2 \mathrm{cpd}$ in E6-MR and E6-HR as a percentage of the flux in E6-XR. Values are averaged over $\pm 10^{\circ}$ of latitude.

\begin{tabular}{lccc}
\hline \hline & \multicolumn{3}{c}{$|k|$} \\
\cline { 2 - 4 } & $0-20$ & $20-60$ & $>60$ \\
\hline E6-MR (\%) & $138 \pm 21$ & $93 \pm 13$ & $3 \pm 2$ \\
E6-HR (\%) & $106 \pm 13$ & $100 \pm 10$ & $31 \pm 17$ \\
E6-XR $\left(10^{2} \mathrm{~kg} \mathrm{~s}^{-2}\right)$ & $63.4 \pm 10.4$ & $21.6 \pm 2.3$ & $5.6 \pm 3.3$ \\
\hline
\end{tabular}

(Andrews et al. 1987). Figure $2 \mathrm{~b}$ shows the vertical EP flux at $80 \mathrm{hPa}$ as a function of planetary wavenumber. We focus on the vertical EP flux, because its vertical divergence dominates the differences of the zonal acceleration between the models, which we discuss below. The divergence of the meridional EP flux imposes considerable forcing too, but this forcing is almost equal in all three model versions. Large-scale equatorial easterly waves with wavenumbers lower than 5 carry most of the meridional EP flux. At these scales, the difference between the model versions is small. The computation of the EP flux in the wavenumber-frequency space follows Horinouchi et al. (2003). The underlying Fourier transform in longitude and time covers each individual month from 2000 to 2003 . The model output has 6-hourly instantaneous values. Table 2 lists the absolutes of the EP flux shown in Fig. 2, integrated over sets of wavenumbers.

Figure $2 \mathrm{~b}$ and Table 2 show that, up to wavenumber 20, the vertical EP flux in the lower stratosphere is largest in E6-MR, the model with the lowest resolution. At wavenumbers smaller than 20, E6-MR has 30\% more EP flux than E6-XR (Table 2). At wavenumbers between 20 and 63, E6-MR and E6-HR still have about the same EP flux as E6-XR. This stands in contrast to the relation of the spectral power of precipitation, which decreases substantially with decreasing spectral resolution at wavenumbers larger than 20, as Table 1 shows. Hence, the quantitative relation of the precipitation variability in the three model versions is not representative for the relationship of the momentum spectrum in the lower stratosphere. Similarly, Lott et al. (2014) and Maury et al. (2013) showed that, despite the pronounced differences in the spectra of tropical precipitation in different GCMs, the spectra of temperature at $80 \mathrm{hPa}$ can be quantitatively similar.

The reason for the quasi independence of the strength of large-scale stratospheric waves from their tropospheric sources in GCMs is still under investigation. Here, we have to clarify the influence of the different tuning of the convective precipitation scheme in E6-MR, E6-HR, and E6-XR on the spectral variability of precipitation, the heating depth (which influences the phase speed distribution of the excited waves), and the

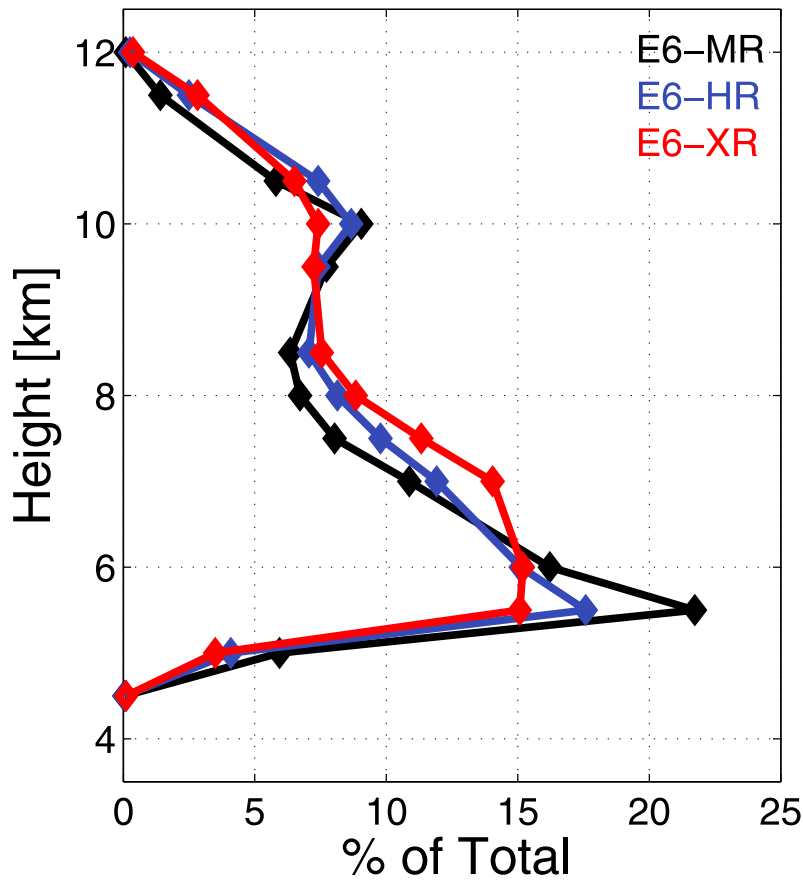

FIG. 3. Probability density function of cloud-top height derived from OLR and temperature, including $\pm 10^{\circ}$ of latitude in E6-MR (black), E6-HR (blue), and E6-XR (red).

EP flux at $80 \mathrm{hPa}$. Therefore, we conducted additional simulations with E6-MR, using the cloud parameters from E6-HR and E6-XR. Thus, we set the mass flux above the nonbuoyancy level and the conversion rate from cloud water to rain in E6-MR from 0.23 and $2 \times$ $10^{-4}$ to 0.23 and $1.5 \times 10^{-4}$ and to 0.16 and $1.2 \times 10^{-4}$, respectively. Set as such, the model does not have a balanced radiation budget and, thus, does not produce a realistic climate (Mauritsen et al. 2012). The simulations thus only cover 3 months.

With the cloud parameters from E6-XR, the spectral variability of precipitation in E6-MR over all resolved wavenumbers is within $5 \%$ of the values obtained with the original setting (not shown). The EP fluxes are within $5 \%$ of the original values up to wavenumber 20 but are $13 \%$ larger at wavenumbers larger than 20 (not shown). These values differ substantially from those listed in Tables 1 and 2 for the spectral precipitation variability and the EP flux in E6-MR, E6-HR, and E6-XR.

The heating depth determines the vertical wavelength and the phase speed of the convectively triggered waves [Ortland et al. (2011), and references therein]. Figure 3 shows the cloud-top height, which is a proxy for heating depth, for E6-MR, E6-HR, and E6-XR. The number of clouds with tops between 4 and $6 \mathrm{~km}$ decreases, and the number of clouds with tops between 6 and $9 \mathrm{~km}$ increases from E6-MR to E6-XR. This is mostly as a result of the changed cloud parameters. As a response to the 
a)

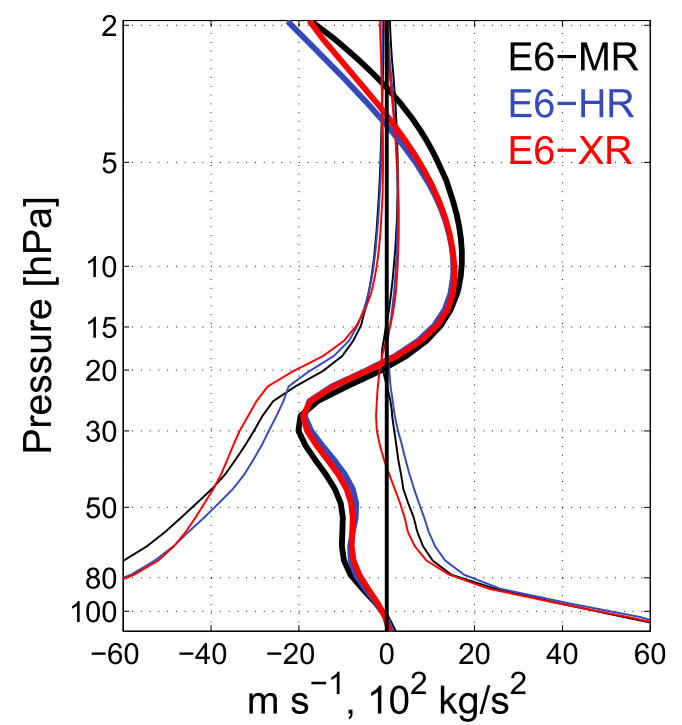

b)

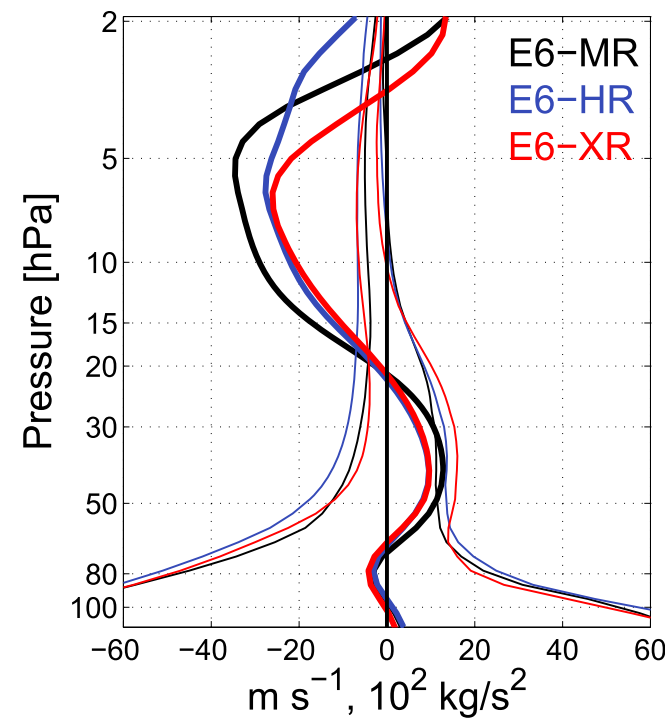

FIG. 4. Profiles of the zonal-mean zonal wind $\left(\mathrm{m} \mathrm{s}^{-1}\right.$; thick lines) and the vertical EP flux $\left(10^{2} \mathrm{~kg} \mathrm{~s}^{-2}\right.$; thin lines) in E6-MR (black), E6-HR (blue), and E6-XR (red) averaged over $\pm 10^{\circ}$ of latitude and all months defined as (a) QBOw and (b) QBOe phase. Lines with positive and negative EP flux below $50 \mathrm{hPa}$ represent waves with easterly and westerly phase speed, respectively.

deepening of the heating within the most abundant clouds, the EP flux at $80 \mathrm{hPa}$ in E6-HR and E6-XR shifts toward higher phase speeds compared to E6-MR; however, the differences amount to only 5\% (not shown).

From the above results, we conclude that the difference of the EP flux in the lowermost stratosphere is mostly because of the influence of spectral resolution on the projection of the convective wave sources on the wave spectrum and the filtering in the tropopause region. Next, we show that the balance of the resolved wave momentum between the three model versions is further altered by differences in the wave damping between the lower stratosphere and the altitude of wave braking.

In the tropical stratosphere, the quasi-biennial oscillation determines the regions where waves meet critical levels and dissipate (Baldwin et al. 2001). Thus, we compare the EP-flux spectra during two well-defined QBO phases, when the influence of the zonal wind on the wave propagation is similar in the three simulations. Figure 4 shows the respective profiles of the zonal-mean zonal wind and the EP flux in E6-MR, E6-HR, and E6-XR. The wind and the EP flux are averages over months when the zonal wind at $20 \mathrm{hPa}$ turns either from westerly to easterly or from easterly to westerly. Thus, the zonal wind shows either a strong westerly or easterly jet at altitudes above $20 \mathrm{hPa}$ and relatively weak flow into the opposite direction at altitudes below $20 \mathrm{hPa}$. For brevity, we refer to the phases with westerly and easterly flow above $20 \mathrm{hPa}$ as QBOw and QBOe, respectively.
Concerning the vertical EP flux, we discuss the quantitative details inherent in Fig. 4 below. Qualitatively, Fig. 4a shows that, during the QBOw phase in all model versions, the wave momentum that westerly waves carry decreases moderately within the easterly jet below $20 \mathrm{hPa}$ and strongly around the onset of the westerly jet at $20 \mathrm{hPa}$. The same holds for easterly waves during the QBOe phase, as Fig. 4b illustrates. The strong divergence of the EP flux around the zero-wind lines at $20 \mathrm{hPa}$ decelerates the jets below $20 \mathrm{hPa}$ and accelerates the jets above. Thus, the forcing causes the upper jets to propagate downward, which is the essential mechanism of the QBO (Holton and Lindzen 1972).

Figure 5 facilitates the quantitative comparison of the EP flux in the lower and the middle stratosphere. The figure shows the vertical EP flux at 80 and $30 \mathrm{hPa}$ during the QBOw and QBOe phases integrated over bins of wavenumbers. The EP fluxes are integrals over equatorial waves with wavenumbers from \pm 1 to \pm 20 , gravity waves resolved in all three model versions with wavenumbers from \pm 20 to \pm 63 , and waves only resolved in E6-HR and E6-XR with wavenumbers larger than \pm 64 . Negative and positive wavenumbers indicate easterly and westerly waves, respectively.

During the QBOw phase, the momentum carried by westerly waves with wavenumbers lower than 20 decreases by about $50 \%$ between 80 and $30 \mathrm{hPa}$, even in the absence of critical levels. Based on E6-MR, we showed in Krismer and Giorgetta (2014) that this is 
a)

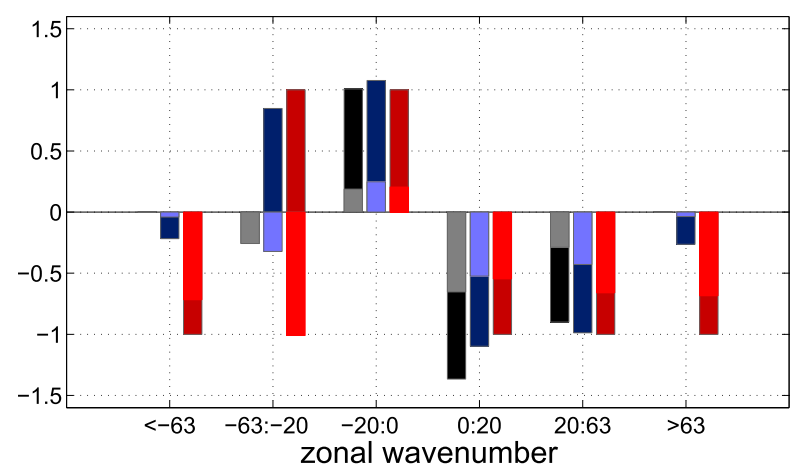

b)

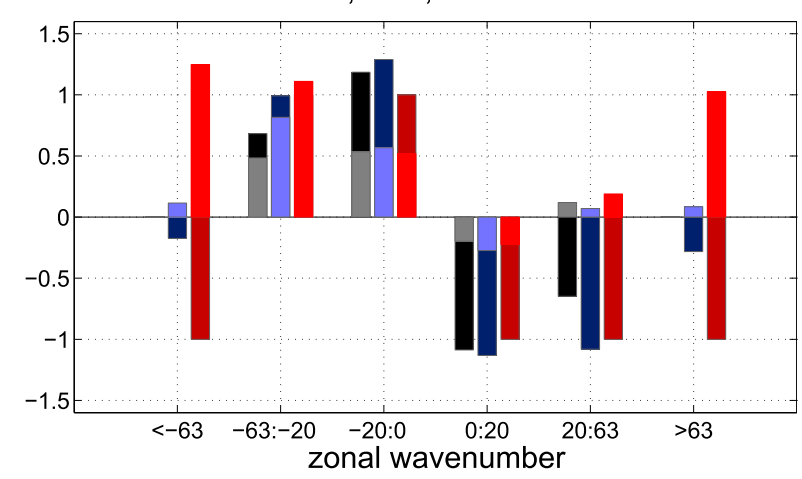

FIG. 5. Normalized vertical EP flux summed over wavenumbers from \pm 0 to \pm 20 , from \pm 21 to \pm 63 , and $> \pm 63$ in E6-MR (black), E6-HR (blue) and E6-XR (red). The fluxes are normalized by the flux in E6-XR at the respective bin of phase speeds. Positive and negative wavenumbers indicate westerly and easterly waves, respectively. Dark and light colored bars show the vertical EP flux at 80 and $30 \mathrm{hPa}$, respectively. The fluxes are averages over months defined as (a) QBO westerly and (b) QBO easterly phase.

mostly caused by radiative wave damping. However, at $30 \mathrm{hPa}$, E6-MR still has the most wave momentum at these wavenumbers. At wavenumbers between +20 and +63 , the wave momentum decreases considerably in E6-MR and E6-HR but diminishes little in E6-XR. Hence, despite the little differences between the model versions above the wave sources at $80 \mathrm{hPa}$ at these scales, the waves carry twice as much momentum in E6-XR as in E6-MR at $30 \mathrm{hPa}$.

During the QBOw phase, most of the easterly waves dissipate at critical levels within the easterly jet in the lower stratosphere. Especially in E6-XR, the EP flux at negative wavenumbers larger than -20 is negative at $30 \mathrm{hPa}$. Doppler-shifted westerly waves, which dominate the wave field after the dissipation of easterly waves, carry this flux. We will show below that these waves contribute substantially to the westerly acceleration of the QBO westerly jet. Figure $5 b$ shows that, during the QBOe phase, the above results apply in reverse order for westerly and easterly waves.

Before we discuss the spectral distribution of the resolved wave forcing in the tropical stratosphere, we investigate the differential wave damping at wavenumbers from 20 to 60 between the lower stratosphere and $30 \mathrm{hPa}$. We find that in ECHAM6 wave damping at large wavenumbers is mostly due to horizontal diffusion (Krismer and Giorgetta 2014). The model applies a horizontal hyperdiffusion scheme where the diffusive tendency is

$$
\frac{\partial \chi_{n}}{\partial t}=-K_{\chi}\left[n(n+1) a^{-2}\right]^{q} \chi_{n}
$$

where $\chi$ is vorticity, divergence, or temperature; $t$ is time; $n$ is the nondimensional wavenumber; $K_{\chi}$ is the constant diffusion coefficient for the respective variable; and $a$ is Earth's radius. A value of $2 q$ is the order of the diffusion scheme. Above the tropopause, $q$ is set to 4 in E6-MR and to 3 in E6-HR and E6-XR. The diffusion coefficient $K_{\chi}$ is given by

$$
K_{\chi}=\frac{1}{\tau}\left[n_{0}\left(n_{0}+1\right) a^{-2}\right]^{-q},
$$

where $\tau$ is the $e$-folding damping time for the highest wavenumber $n_{0}$ and is set to $7,1.5$, and $0.5 \mathrm{~h}$ in E6-MR, E6-HR, and E6-XR, respectively. The highest wavenumber $n_{0}$ in E6-MR, E6-HR, and E6-XR is 63, 127, and 255, respectively. Figure 6 shows the evaluation of Eq. (1) for the spectral components of the zonal-mean zonal wind $u_{n}$ at $80 \mathrm{hPa}$ in E6-MR, E6-HR, and E6-XR. In E6-MR and E6-HR, horizontal diffusion strongly acts on waves with wavenumbers larger than 20 . The diffusive dissipation of these waves causes the decrease of wave momentum between the wave sources in the lower stratosphere and $30 \mathrm{hPa}$ shown in Fig. 5. In E6-XR,

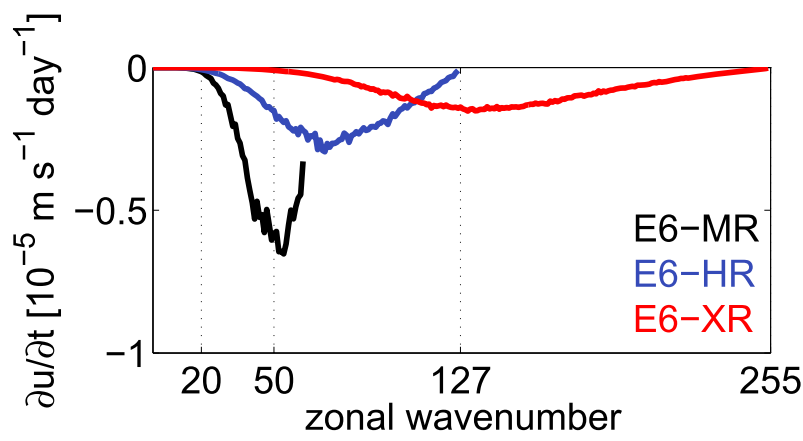

FIG. 6. Tendency of the zonal-mean zonal wind because of horizontal diffusion at $80 \mathrm{hPa}\left(10^{-5} \mathrm{~m} \mathrm{~s}^{-1}\right.$ day $\left.^{-1}\right)$ as a function of the zonal wavenumber in E6-MR (black), E6-HR (blue), and E6-XR (red) averaged over $\pm 10^{\circ}$. 
a)

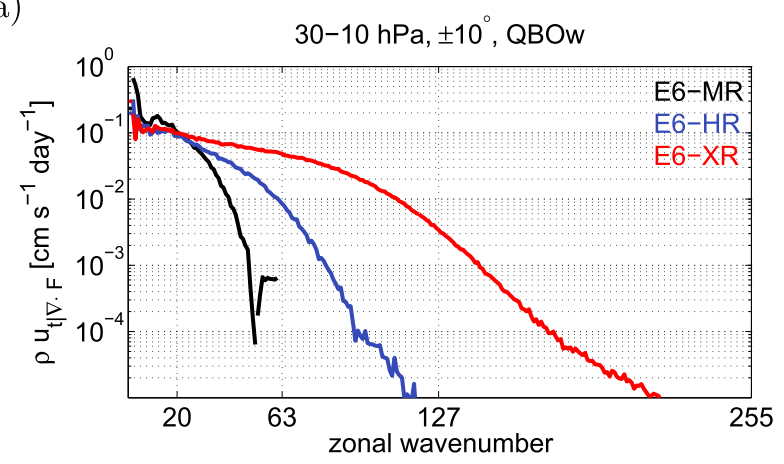

c)

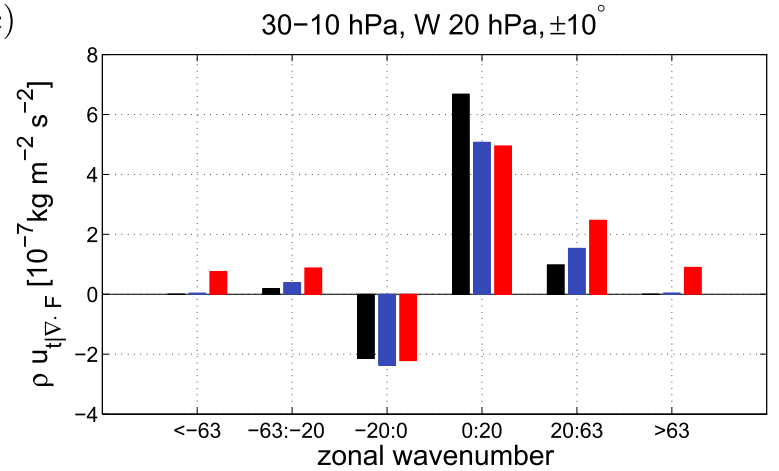

b)

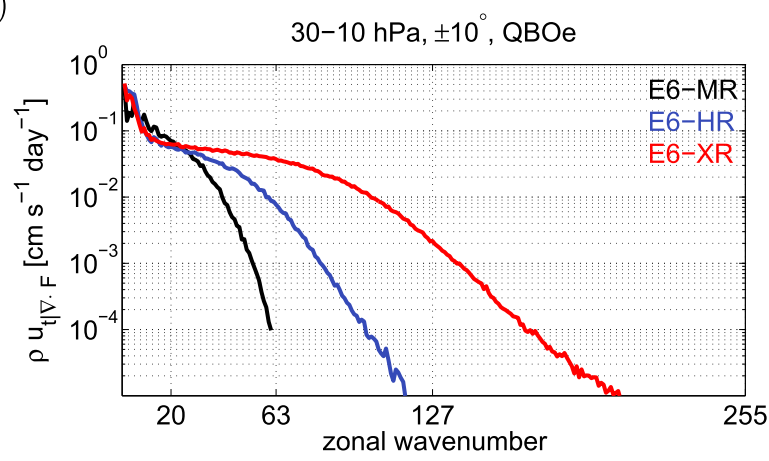

d)

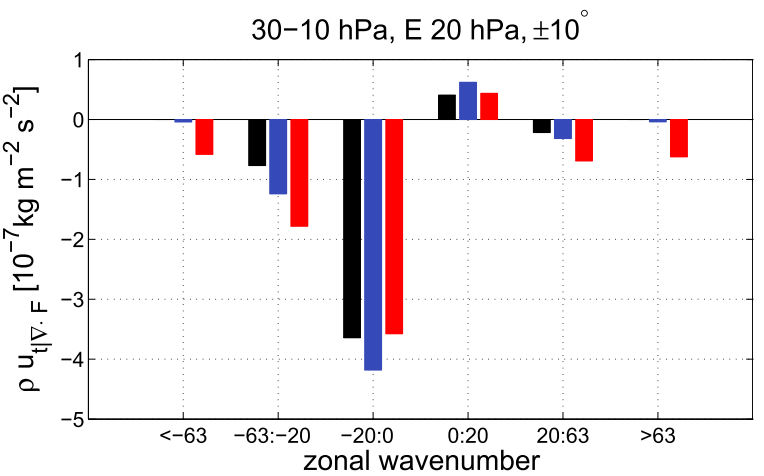

FIG. 7. (a),(b) The density-corrected resolved wave forcing because of the divergence of the horizontal and vertical EP flux summed from 30 to $10 \mathrm{hPa}$ over $\pm 10^{\circ}$ of latitude as a function of the zonal wavenumber in E6-MR (black), E6-HR (blue), and E6-XR (red) for values integrated over easterly and westerly waves, respectively. (c),(d) A quantitative comparison showing the integrals of the respective forcing over waves with wavenumbers from \pm 1 to \pm 20 , from \pm 20 to \pm 63 , and $> \pm 63$. The forcing is the average over all months defined as (a),(c) QBOw and (b),(d) QBOe phase.

horizontal diffusion slowly increases after wavenumber 50. Hence, as Fig. 5 shows, waves with wavenumbers between 20 and 63 lose relatively little vertical EP flux in the absence of critical levels in E6-XR. The horizontal diffusion decreases with wavenumber at wavenumbers larger than 50, 70, and 140 in E6-MR, E6-HR, and E6-XR, respectively. According to Eq. (1), the horizontal diffusion depends linearly on the amplitude of the waves. Apparently, at high wavenumbers, the decrease of amplitude with wavenumber overcompensates for the increase of the term in brackets in Eq. (1).

\section{c. Forcing of the mean flow}

Figure 4a shows that the negative vertical EP flux that westerly waves carry strongly decreases between 30 and $10 \mathrm{hPa}$. The same is valid for the positive EP flux that easterly waves carry in Fig. 4b. The divergence of the EP flux forces the mean flow. It decelerates the jets below $20 \mathrm{hPa}$, and accelerates the jets above. The forcing causes the upper jets to propagate downward (Holton and Lindzen 1972).
Figures $7 \mathrm{a}$ and $7 \mathrm{~b}$ show the density-corrected resolved wave forcing because of the divergence of the horizontal and vertical EP flux summed from 30 to $10 \mathrm{hPa}$ in the three model versions as a function of the zonal wavenumber. For a quantitative comparison, Figs. $7 \mathrm{c}$ and $7 \mathrm{~d}$ show the integrals of the respective forcing over waves with wavenumbers from \pm 1 to \pm 20 , from \pm 20 to \pm 63 , and larger than \pm 63 . The selected altitude range corresponds to the region of strong westerly and easterly forcing around the zero-wind line between the QBO jets. Figures $7 \mathrm{a}$ and $7 \mathrm{c}$ illustrate three points: First, during the QBOw phase, from wavenumber 1 to 20 the wave forcing is strongest in E6-MR and is equally strong in E6-HR and E6-XR. Second, the forcing generally decreases with increasing wavenumber at rates that decrease with increasing spectral resolution. Thus, at wavenumbers between 20 and 63 and between 20 and 127, the wave forcing increases with spectral resolution, is weakest in E6-MR, is intermediate in E6-HR, and is strongest in E6XR. Third, naturally the spectrum widens with increasing spectral resolution. Figure $7 \mathrm{c}$ shows that easterly waves with wavenumbers lower than 20 cancel a substantial 
amount of the westerly forcing. These waves penetrate deep into the easterly jet and dissipate above its maximum at $30 \mathrm{hPa}$. Doppler-shifted westerly waves with negative wavenumbers larger than 20 contribute small westerly forcing. These results also hold for the QBOe phase in reverse order for westerly and easterly waves, as shown in Figs. 7b and 7d. However, the forcing caused by waves with wavenumbers between 1 and 20 is about equally strong in all three model versions because they have about equal vertical EP fluxes, both in the lower stratosphere and at $30 \mathrm{hPa}$, as shown in Fig. 5.

\section{Summary and conclusions}

In this study, we show how the spectral apportionment of the resolved tropical wave activity depends on the spectral truncation of the atmospheric general circulation model ECHAM6. Therefore, we perform AMIP-type simulations with spectral truncations of T63 (E6-MR), T127 (E6-HR), and T255 (E6-XR). To limit the biases of global temperature, precipitation, and wind, the three model versions have adjusted tuning parameters for convection, the strength of parameterized gravity waves, and the efficiency of the implicit numerical diffusion scheme. Tests show that the effects of these adjustments are negligible compared to the effect of higher resolution.

We find that the strength of the tropospheric wave sources increases with spectral resolution at wavenumbers larger than 20 , even at scales resolved well by the T63 version. However, the momentum waves carry in the lower stratosphere decreases with spectral resolution at wavenumbers smaller than 20 and remains approximately stable at wavenumbers between 20 and 50. The differences of the wave sources in the three model versions might be cancelled out by different coupling between the wave sources and the freely propagating waves, as well as tropospheric wave filtering. In the stratosphere, longwave radiative damping and horizontal diffusion attenuate the upwardpropagating waves. The scale-selective horizontal hyperdiffusion scheme of ECHAM6 acts on larger wavenumbers with decreasing resolution, and there is substantial wave damping in the T63 version at wavenumbers larger than 20 , which are still unaffected in the higher-resolution versions.

Most of today's GCMs use gravity wave parameterizations to transport sufficient momentum into the stratosphere. Our comparison of the resolved wave forcing shows that these parameterizations have to account not only for the drag imposed by unresolved waves, but also for the lack of momentum at scales that are represented insufficiently in low-resolution models.
Further, we show that even the high-resolution model version truncated at $\mathrm{T} 255$ lacks precipitation variability at wavenumbers larger than 50 when compared to TRMM observations. At these scales, increasing spectral precipitation variability leads to more vertical EP flux in the lower stratosphere. Hence, to simulate stratospheric waves in GCMs realistically, modelers have to improve the convective parameterization to better represent the variability of mesoscale precipitation.

Acknowledgments. We acknowledge the MPI-ESM modelling group for technical support. We thank Elisa Manzini for fruitful discussions and Eileen Dahms for technical support. Computational resources to conduct the AMIP experiments were made available by Deutsches Klimarechenzentrum (DKRZ) through support from the Bundesministerium für Bildung und Forschung (BMBF) for the CMIP5 and STORM consortium projects.

\section{REFERENCES}

Alexander, M. J., P. T. May, and J. H. Beres, 2004: Gravity waves generated by convection in the Darwin area during the Darwin Area Wave Experiment. J. Geophys. Res., 109, D20S04, doi:10.1029/2004JD004729.

Andrews, D. G., J. R. Holton, and C. B. Leovy, 1987: Middle Atmosphere Dynamics. International Geophysics Series, Vol. 40, Academic Press, 489 pp.

Baldwin, M. P., and Coauthors, 2001: The quasi-biennial oscillation. Rev. Geophys., 39, 179-229, doi:10.1029/1999RG000073.

Bergman, J. W., and M. L. Salby, 1994: Equatorial wave activity derived from fluctuations in observed convection. J. Atmos. Sci., 51, 3791-3806, doi:10.1175/1520-0469(1994)051<3791: EWADFF $>2.0 . \mathrm{CO} ; 2$.

Cohen, N., E. Gerber, and O. Bühler, 2014: What drives the Brewer-Dobson circulation? J. Atmos. Sci., 71, 3837-3855, doi:10.1175/JAS-D-14-0021.1.

Crueger, T., C. Hohenegger, and W. May, 2013: Tropical precipitation and convection changes in the Max Planck Institute Earth system model (MPI-ESM) in response to $\mathrm{CO}_{2}$ forcing. J. Adv. Model. Earth Syst., 5, 85-97, doi:10.1002/jame.20012.

Dunkerton, T. J., 1997: The role of gravity waves in the quasibiennial oscillation. J. Geophys. Res., 102, 26053-26076, doi:10.1029/96JD02999.

, and M. P. Baldwin, 1991: Quasi-biennial modulation of planetary-wave fluxes in the Northern Hemisphere winter. $J$. Atmos. Sci., 48, 1043-1061, doi:10.1175/1520-0469(1991)048<1043: QBMOPW $>2.0 . \mathrm{CO} ; 2$.

Ern, M., and P. Preusse, 2009: Quantification of the contribution of equatorial Kelvin waves to the QBO wind reversal in the stratosphere. Geophys. Res. Lett., 36, L21801, doi:10.1029/ 2009GL040493.

, H.-K. Cho, P. Preusse, and S. D. Eckermann, 2009: Properties of the average distribution of equatorial Kelvin waves investigated with the GROGRAT ray tracer. Atmos. Chem. Phys., 9, 7973-7995, doi:10.5194/acp-9-7973-2009.

, and Coauthors, 2014: Interaction of gravity waves with the QBO: A satellite perspective. J. Geophys. Res. Atmos., 119, 2329-2355, doi:10.1002/2013JD020731. 
Evan, S., M. J. Alexander, and J. Dudhia, 2012: WRF simulations of convectively generated gravity waves in opposite QBO phases. J. Geophys. Res., 117, D12117, doi:10.1029/2011JD017302.

Fritts, D., and M. J. Alexander, 2003: Gravity wave dynamics and effects in the middle atmosphere. Rev. Geophys., 41, 1003, doi:10.1029/2001RG000106.

Gates, W. L., 1992: AMIP: The Atmospheric Model Intercomparison Project. Bull. Amer. Meteor. Soc., 73, 1962-1970, doi:10.1175/1520-0477(1992)073<1962:ATAMIP>2.0.CO;2.

Giorgetta, M. A., E. Manzini, E. Roeckner, M. Esch, and L. Bengtsson, 2006: Climatology and forcing of the quasibiennial oscillation in the MAECHAM5 model. J. Climate, 19, 3882-3901, doi:10.1175/JCLI3830.1.

, and Coauthors, 2012: CMIP5 simulations of the Max Planck Institute for Meteorology (MPI-M) based on the MPI-ESM-MR model: The AMIP experiment. World Data Center for Climate, accessed 5 December 2014, doi:10.1594/WDCC/CMIP5. MXMRam.

—-, and Coauthors, 2013: Climate and carbon cycle changes from 1850 to 2100 in MPI-ESM simulations for the Coupled Model Intercomparison Project phase 5. J. Adv. Model. Earth Syst., 5, 572-597, doi:10.1002/jame.20038.

Hertwig, E., J. S. von Storch, D. Handorf, K. Dethloff, I. Fast, and T. R. Krismer, 2014: Effect of horizontal resolution on ECHAM6-AMIP performance. Climate Dyn., 45, 185-211, doi:10.1007/s00382-014-2396-x.

Hines, C. O., 1997a: Doppler-spread parameterization of gravitywave momentum deposition in the middle atmosphere. Part 1: Basic formulation. J. Atmos. Sol.-Terr. Phys., 59, 371-386, doi:10.1016/S1364-6826(96)00079-X.

_ $1997 \mathrm{~b}$ : Doppler-spread parameterization of gravity-wave momentum deposition in the middle atmosphere. Part 2: Broad and quasi monochromatic spectra, and implementation. J. Atmos. Sol.-Terr. Phys., 59, 387-400, doi:10.1016/S1364-6826(96)00080-6.

Holton, J., and R. Lindzen, 1972: An updated theory for the quasi-biennial cycle of the tropical stratosphere. J. Atmos. Sci., 29, 1076-1080, doi:10.1175/1520-0469(1972)029<1076: AUTFTQ $>2.0 . \mathrm{CO} ; 2$.

Horinouchi, T., and Coauthors, 2003: Tropical cumulus convection and upward-propagating waves in middle-atmospheric GCMs. J. Atmos. Sci., 60, 2765-2782, doi:10.1175/ 1520-0469(2003)060<2765:TCCAUW >2.0.CO;2.

Huffman, G. J., and Coauthors, 2007: The TRMM Multisatellite Precipitation Analysis (TMPA): Quasi-global, multiyear, combined-sensor precipitation estimates at fine scales. J. Hydrometeor., 8, 38-55, doi:10.1175/JHM560.1.

Kawatani, Y., S. Watanabe, K. Sato, T. J. Dunkerton, S. Miyahara, and M. Takahashi, 2010: The roles of equatorial trapped waves and internal inertia-gravity waves in driving the quasi-biennial oscillation. Part I: Zonal mean wave forcing. J. Atmos. Sci., 67, 963-980, doi:10.1175/2009JAS3222.1.

Krismer, T. R., and M. A. Giorgetta, 2014: Wave forcing of the quasibiennial oscillation in the Max Planck Institute Earth System Model. J. Atmos. Sci., 71, 1985-2006, doi:10.1175/JAS-D-13-0310.1. - - , and M. Esch, 2013: Seasonal aspects of the quasibiennial oscillation in the Max Planck Institute Earth System Model and ERA-40. J. Adv. Model. Earth Syst., 5, 406-421, doi:10.1002/jame.20024.

Lott, F., 1999: Alleviation of stationary biases in a GCM through a mountain drag parameterization scheme and a simple representation of mountain lift forces. Mon. Wea. Rev., 127, 788-801, doi:10.1175/1520-0493(1999)127<0788: AOSBIA $>2.0 . \mathrm{CO} ; 2$.
— , and Coauthors, 2014: Kelvin and Rossby-gravity wave packets in the lower stratosphere of some high-top CMIP5 models. J. Geophys. Res. Atmos., 119, 2156-2173, doi:10.1002/ 2013JD020797.

Manzini, E., and N. A. McFarlane, 1998: The effect of varying the source spectrum of a gravity wave parameterization in a middle atmosphere general circulation model. J. Geophys. Res., 103, 31 523-31 539, doi:10.1029/98JD02274.

_,- _ and C. McLandress, 1997: Impact of the Doppler spread parameterization on the simulation of the middle atmosphere circulation using the MA/ECHAM4 general circulation model. J. Geophys. Res., 102, 25 751-25 762, doi:10.1029/97JD01096.

—, M. A. Giorgetta, M. Esch, L. Kornblueh, and E. Roeckner, 2006: The influence of sea surface temperatures on the northern winter stratosphere: Ensemble simulations with the MAECHAM5 model. J. Climate, 19, 3863-3881, doi:10.1175/JCLI3826.1.

Mauritsen, T., and Coauthors, 2012: Tuning the climate of a global model. J. Adv. Model. Earth Syst., 4, M00A01, doi:10.1029/ 2012MS000154.

Maury, P., F. Lott, L. Guez, and J.-P. Duvel, 2013: Tropical variability and stratospheric equatorial waves in the IPSLCM5 model. Climate Dyn., 40, 2331-2344, doi:10.1007/s00382-011-1273-0.

NASA, 2014:TRMM_3B42_daily version 6: Daily TRMM and other satellites precipitation product (3B42 V6 derived). Goddard Space Flight Center Distributed Active Archive Center, accessed April 2014. [Available online at http://disc.sci.gsfc.nasa.gov/ datacollection/TRMM_3B42_daily_V6.html.]

Ortland, D. A., M. J. Alexander, and A. W. Grimsdell, 2011: On the wave spectrum generated by tropical heating. J. Atmos. Sci., 68, 2042-2060, doi:10.1175/2011JAS3718.1.

Salby, M. L., and R. R. Garcia, 1987: Transient response to localized episodic heating in the tropics. Part I: Excitation and short-time near-field behavior. J. Atmos. Sci., 44, 458-498, doi:10.1175/1520-0469(1987)044<0458:TRTLEH>2.0.CO;2.

Sato, K., and T. J. Dunkerton, 1997: Estimates of momentum flux associated with equatorial Kelvin and gravity waves. J. Geophys. Res., 102, 26 247-26261, doi:10.1029/96JD02514.

Scaife, A. A., N. Butchart, C. D. Warner, D. Stainforth, W. Norton, and J. Austin, 2000: Realistic quasi-biennial oscillations in a simulation of the global climate. Geophys. Res. Lett., 27, 34813484, doi:10.1029/2000GL011625.

Schmidt, H., and Coauthors, 2013: Response of the middle atmosphere to anthropogenic and natural forcings in the CMIP5 simulations with the Max Planck Institute Earth system model. J. Adv. Model. Earth Syst., 5, 98-116, doi:10.1002/jame.20014.

Stevens, B., and Coauthors, 2013: Atmospheric component of the MPI-M earth system model: ECHAM6. J. Adv. Model. Earth Syst., 5, 146-172, doi:10.1002/jame.20015.

Takahashi, M., 1999: Simulation of the Quasi-Biennial Oscillation in a general circulation model. Geophys. Res. Lett., 26, 13071310, doi:10.1029/1999GL900188.

Tomassini, L., E. P. Gerber, M. P. Baldwin, F. Bunzel, and M. Giorgetta, 2012: The role of stratosphere-troposphere coupling in the occurrence of extreme winter cold spells over northern Europe. J. Adv. Model. Earth Syst., 4, M00A03, doi:10.1029/2012MS000177.

Yanai, M., and T. Maruyama, 1966: Stratospheric wave disturbances propagating over the equatorial Pacific. J. Meteor. Soc. Japan, 44, 291-294.

Zhu, X., 1993: Radiative damping revisited: Parameterization of damping rate in the middle atmosphere. J. Atmos. Sci., 50, 3008-3021, doi:10.1175/1520-0469(1993)050<3008: RDRPOD $>2.0 . \mathrm{CO} ; 2$. 